\title{
Bacteriological profile in UTI Cases with special reference to Amikacin sensitivity in a tertiary Care centre in Western UP
}

\author{
Ritu Agarwal ${ }^{1, *}$, Gunjan Pandey ${ }^{2}$ \\ ${ }^{1}$ Associate Professor, ${ }^{2}$ Tutor, Dept. of Microbiology, G. S. Medical College \& Hospital, Hapur, Uttar Pradesh, India
}

*Corresponding Author:

Email: ritu15agarwal@gmail.com

\begin{abstract}
Introduction: UTI is the most common bacterial infection worldwide with women in reproductive age group being 30 times more likely to suffer from it than men due to short urethra and proximity of uretheral opening with anal canal. Escherichia coli is the most common organism responsible for UTI and there has been an increase in incidence of community acquired UTI caused by ESBL producing E. coli. This study was planned to see the sensitivity of amikacin in urinary isolates so it can be considered as a treatment option in ESBL producing organisms, reserving high-end drugs like carbapenems.

Materials and Methods: The retrospective study was carried out between January 2017 to August 2017 with 1025 patients of suspected UTI being screened for growth of bacteria in their urine samples and their sensitivity to antibiotics. Both males and females were included in the study, ages ranging from 0 year to $>80$ yrs. Urine samples were inoculated on Blood agar and MacConkey agar. All positive cultures were tested for antibiotic susceptibility testing by Kirby bauer disc diffusion technique.

Results: Out of total 1025 samples, growth was detected in 171 (16.7\%) samples. Out of these $121(70.8 \%)$ were females, and 50 (29.2\%) were males. Maximum number of males are seen in age group 21-30yrs i.e. 13 (26\%) followed by 41 to $50 \mathrm{yrs}$ i.e. 12 (24\%). Also, maximum number of females are seen in age group 21-30yrs i.e. 41 (33.9\%) followed by 21 (17.4\%) patients in age group 41-50yrs. Most common microbial agent isolated was Esch.coli (97/171), followed by Klebsiella spp. (25/171). The important finding of the study was the sensitivity pattern of amikacin which was found to be sensitive in $61 \%$ of the isolates which is quite significant and we can use amikacin rather than high end antibiotics like carbapenems in these cases of UTI which will prevent emergence of resistance against them.

Conclusion: Our finding i.e. Amikacin being highly sensitive to urinary pathogens reflects upon the facts that in sensitive cases, Amikacin can be used for ESBL producing uropathogens instead of costly high end antibiotics like Carbapenams.
\end{abstract}

Keywords: UTI, ESBL producing E. coli, Amikacin.

\section{Introduction}

UTI is the cause of major share of patients presenting to physicians and also the reason of maximum number of urine samples received in microbiology laboratory for culture and sensitivity. ${ }^{1}$ In general, females are affected more than males and almost $40 \%$ of women develop UTI at some point in their life. Adult women are 30 times more likely than men to develop a UTI, with almost half of them experiencing at least one episode of UTI during their lifetime. ${ }^{2}$ because of short urethra, close proximity to anus and sexual activity. Other susceptible adults include the elderly and patients requiring urethral catheterization. Amongst all the gram negative bacilli and gram positive cocci causing UTI, E. coli is the most common cause of UTI worldwide, ${ }^{3,4}$ and there has been an increase in the incidence of community acquired UTI caused by ESBL producing Esch.coli. ${ }^{5,6}$ The treatment options for such ESBL producing Esch.coli ${ }^{7}$ are very limited especially in outpatient settings as ESBL are enzymes which cause degradation of most of $\beta$ lactam antibiotics viz. penicillin and cephalosporins. Carbapenems are the drug of choice for infections caused by ESBL producing pathogens however there are a host of drawbacks associated with carbapenems viz high cost, hospitalization required (except for ertapenem) and development of resistance against them by their frequent usage. Other options include combination of $\beta$ lactam and $\beta$ lactamase inhibitor, $4^{\text {th }}$ generation cephalosporins, tigecycline, nitrofurantoin, fosfomycin etc.

Over the last decade there has been a renewed interest in the therapeutic role of aminoglycosides viz amikacin in the treatment of mild to moderate UTI caused by ESBL producing Esch.coli. Amikacin definitely shows good in vitro sensitivity in ESBL positive organisms ${ }^{8}$ and it attains a high concentration in urine so therapeutic value is promising in treatment of UTI. Also low cost and once daily dosing make it a good treatment option for pathogens showing sensitivity to amikacin causing mild to severe UTI. The only limiting factor in usage of amikacin is its nephrotoxicity and ototoxicity for which the patients have to be closely monitored for renal parameters and auditory symptoms. There have been studies on amikacin OPAT (outpatient parenteral antibiotic therapy) in children and females suffering from UTI and they show promising results. ${ }^{9}$

This study was planned keeping in mind that ESBL producing Esch.coli and GNBs causing UTI are on a rise and so an estimate of amikacin sensitivity in this area could guide treatment for such cases and instead of carbapenams, amikacin can be used in such patients. 
This way we can reserve carbapenems for severe cases and also prevent development of resistance against it.

\section{Materials and Methods}

This retrospective study was conducted in a peripheral tertiary health care centre in UP and data on urine culture and sensitivity was collected from January 2017 to August 2017. Information on microorganisms isolated from urine samples of suspected UTI patients who had presented to OPD with sign and symptoms of UTI viz. fever, frequency, burning micturition was collected and analyzed. The patients admitted in wards that were either admitted for the UTI symptoms or for some other condition but developed UTI in course of time were also included in the study.

The urine samples were processed according to standard guidelines. ${ }^{10}$ The urine samples were inoculated on Blood agar and MacConkey agar by the semi quantitative plating method using the calibrated loop technique $(0.001 \mathrm{ml})$. After $18-24$ hours of aerobic incubation, the growth was subjected to identification of the organism by standard biochemicals and antibiotic sensitivity testing was done by Kirby bauer disc diffusion technique according to CDC guidelines .Urine culture reports were carefully collected, observed and analyzed for the organism isolated, its sensitivity pattern to various antibiotics besides the demographic data. Patients from all age groups, sex with symptomatic bacteriuria were included in the study and those patients who had taken antibiotic with in previous 30 days of culture were excluded from the study.

\section{Results}

The total number of urine samples received in microbiology lab from January 2017 to August 2017 was 1025 , out of which growth was seen in 171 samples (16.7\%) table 1.

Table 1: Urine samples showing growth and no growth after culture

\begin{tabular}{|l|l|c|}
\hline S. No. & Particulars & Value \\
\hline $\mathbf{1}$ & Total Cases & 1025 \\
\hline $\mathbf{2}$ & Growth & 171 \\
\hline $\mathbf{3}$ & Sterile & 854 \\
\hline
\end{tabular}

Out of 171 culture positive cases, females outnumbered males as 121 female samples were culture positive as compared to that of 50 males, Graph 1.

\section{Chart 1: Distribution of growth positive cases with respect to sex}

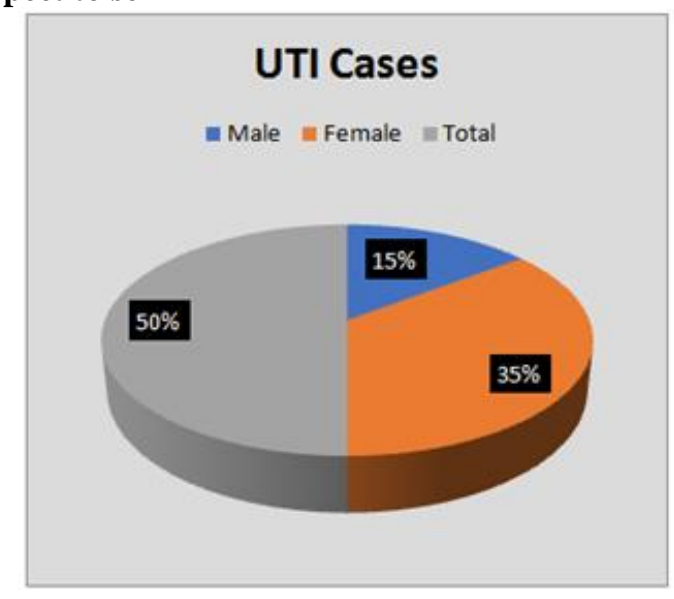

Amongst the culture positive samples maximum number of males were seen in the age group 21 to 30 years i.e. $13 / 50(26 \%)$ followed by $24 \%$ in the 41 to 50 years age group. In females also maximum patients were in the 21 to 30 years age group i.e. 41 out of $121(33.9 \%)$ followed by 21 patients out of $121(17.4 \%)$ in the 41-50 year age group. There was no sample from any patient above 80 years of age whereas 2 males and 1 female presented in the 0 to 10 year age group, Graph 2.

Esch.coli was the most common organism isolated 97/171 (56.7\%) from the samples followed by Klebsiella species 25/171(14.6\%). Staphylococcus aureus was seen in 15 cases (8.8\%) whereas coagulase negative Staphylococcus(CONS) species were found in 12 cases (7\%). Enterobacter species and Proteus species were seen in 5 samples each whereas Pseudomonas species and Citrobacter species was seen in $1.8 \%$ samples. Acinetobacter was seen in 2 cases whereas Candida species were seen in 4 samples Table 2 . 


\section{Chart 2: Distribution of growth positive cases with respect to age and gender}

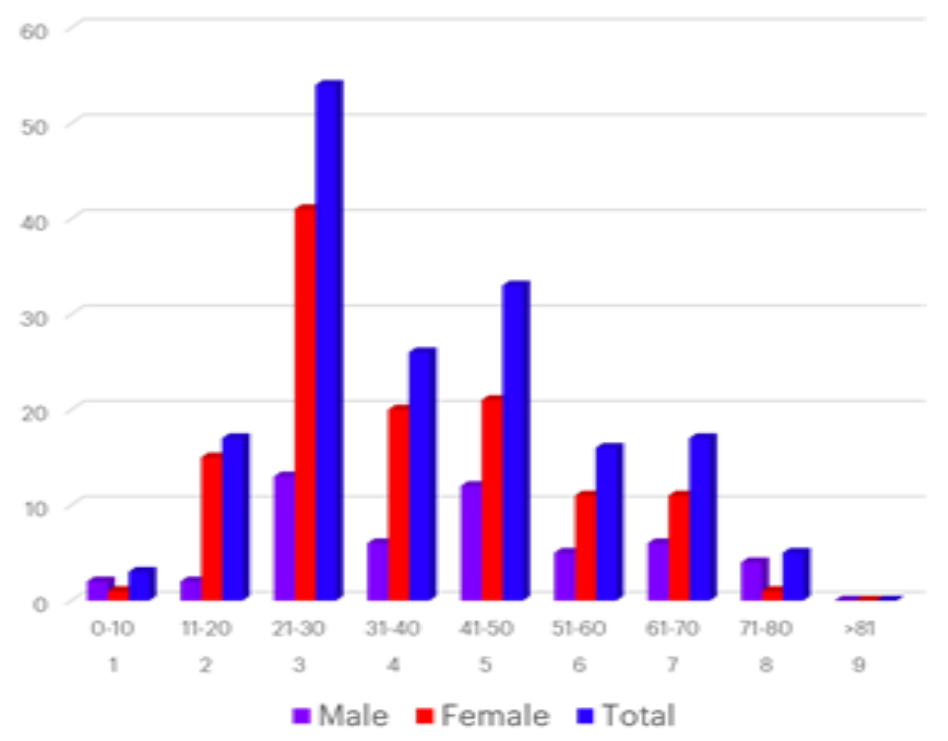

Table 2: Spectrum of bacterial isolates obtained from culture positive cases

\begin{tabular}{|c|l|c|}
\hline S. No. & Particular & Value \\
\hline 1 & Esch.coli & 97 \\
\hline 2 & Klebsiella & 25 \\
\hline 3 & Pseudomonas & 3 \\
\hline 4 & Citrobacter & 3 \\
\hline 5 & CONS & 12 \\
\hline 6 & S.Aureus & 15 \\
\hline 7 & Acinetobacter & 2 \\
\hline 8 & Enterobacter & 5 \\
\hline 9 & Proteus & 5 \\
\hline 10 & Candida Spp. & 4 \\
\hline
\end{tabular}

During antimicrobial susceptibility testing, amikacin was found to be sensitive in 104 out of 171 cases $(61 \%)$ whereas gentamicin was found to be sensitive in $26 \%$ cases, Graph 3.

\section{Graph 3: Sensitivity pattern of organisms to Amikacin}

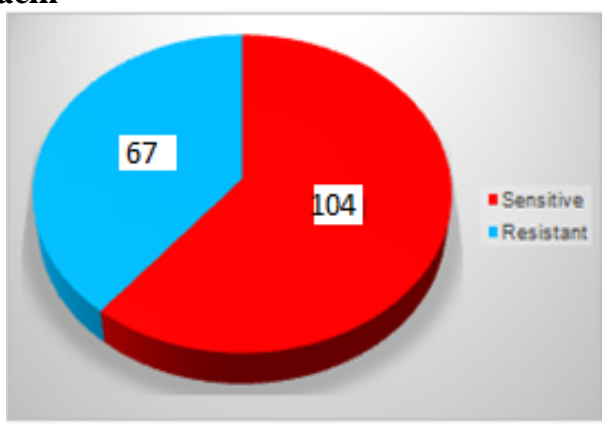

\section{Discussion}

More number of females as compared to males suffering from UTI as we found in our study have also been seen in studies by Harish et al and Subedi et al. ${ }^{11,12}$
Subedi et al found in their study that $84.8 \%$ of total cases of UTI were females while our study shows that $71 \%$ of cases were from female population. The most probable reason for the above findings is short urethra and its close proximity to anus which makes them more vulnerable to UTI.

Maximum number of males (26\%) and females (33.9\%) falling in 21-30 years age group coincides with the study of Subedi et al and Harish et al. ${ }^{11,12}$ Harish et al stated in their study that $38.29 \%$ of patients were in the 21-29 year age group while our study shows $31.6 \%(54 / 171)$ patients in the same age group. The reason for above findings could be increased physical and sexual activity in this age group.

The distribution of organisms recovered from UTI cases confirms with the study of Beyene et al. Their study reveals Esch.coli was isolated from 50\% culture positive cases while our study shows $56.7 \%$ isolation of Esch.coli from urine samples of UTI patients. ${ }^{13}$ Our study shows amikacin sensitivity to be 104/171 (60.8\%) which is much in agreement with study of $\mathrm{R}$ nalini et al ${ }^{14}$ whose study depicts amikacin sensitivity to be $66.5 \%$ and reflects. A study by Shalini et al shows amikacin sensitivity at a good $98.9 \%$ which reflects presence of good treatment options apart from carbapenems ${ }^{15}$. The study by Singhal et al ${ }^{16}$ shows amikacin sensitivity to be $72 \%$ in IPD patients while $89 \%$ in OPD patients. Overall amikacin is still a much countable option in treatment of UTI caused by ESBL producing Esch.coli.

The mechanism of action of amikacin, its pharmacokinetic and pharmacodynamic make ita relevant substitute for carbapenams. An animal study shows that urine concentration of amikacin remains above MIC for approx 4 days after the last dose and so then is considerable post antibiotic coverage. 


\section{Conclusion}

The magnitude of problem of incidence of UTI, developing resistance in microorganisms causing UTI and changes in sensitivity patterns of microorganisms towards antibiotics causes this issue to be looked upon time and again. Need for timely revision of antibiotic sensitivity pattern and empirical therapy of UTI according to the current behavior of microorganisms is ever dwelling. Age old drug like amikacin presents a good treatment option for mild to moderate UTI cases caused by ESBL producing uropathogens. Since no new molecules are seen coming in near future, judicious use of existing drugs is the best way to navigate the current situation.

Funding: No funding sources.

Conflict of interest: None declared.

\section{References}

1. Stamm WE. The epidemiology of urinary tract infections: Risk factors reconsidered. Inter Sci Conf Antimicrob Agents Chemother 1999;39:769.

2. Foxman B. Epidemiology of urinary tract infections: incidence, morbidity, and economic costs. Am J Med 2002;113(Suppl 1A):5S-13S. [PubMed].

3. Behzadi P, Behzadi E, Yazdanbod H, Aghapour R, Akbari Cheshmeh M, Salehian Omran D. A survey on urinary tract infections associated with the three most common uropathogenic bacteria 2010;5(2):111115.PMID: 21977133.

4. Ritu Agarwal, Maneesh Goyal, Dakshina Bisht, Rinku Garg. Bacterial Isolates and their Antibiotic Sensitivity Profile Recovered from Urine Samples in NCR, Ghaziabad (Uttar Pradesh). J Evolution of 2014/ Med Dent Sci 2014;3(28):7831-36. DOI: 10.14260/jemds/2980

5. Picozzi SCM, Casellato S, Rossini M, et al. Extendedspectrum beta-lactamase-positive Escherichia coli causing complicated upper urinary tract infection: Urologist should act in time. Urol Ann 2014;6(2):107-12. doi:10.4103/0974-7796.130536.

6. Toner, L., Papa, N., Aliyu, S.H. et al.Extended-spectrum beta-lactamase-producing Enterobacteriaceae in hospital urinary tract infections: incidence and antibiotic susceptibility profile over 9 years. World J Urol 2016;34:1031. https://doi.org/10.1007/s00345-0151718x.

7. Pana ZD and Zaoutis T. Treatment of extendedspectrum $\beta$-lactamase-producing Enterobacteriaceae
(ESBLs) infections: what have we learned until now? [version 1; referees: 2 approved]. F1000Research 2018, 7(F1000 Faculty Rev):1347 (https://doi.org/10.12688/f1000research.14822.1)

8. Cho S-Y, Choi S-M, Park SH, Lee D-G, Choi J-H, Yoo J$\mathrm{H}$. Amikacin therapy for urinary tract infections caused by extended-spectrum $\beta$-lactamase-producing Escherichia coli. Korean J Internal Med 2016;31(1):15661. doi:10.3904/kjim.2016.31.1.156.

9. Polat, Meltem, MD; Tapisiz, Anil, MD. Amikacin Monotherapy for Treatment of Febrile Urinary Tract Infection Caused by Extended-Spectrum $\beta$-Lactamaseproducing Escherichia coli in Children. Pediatr Infectious Dis J 2018;37(4):378-79.doi: 10.1097/INF.0000000000001860.

10. Mackie \& McCartney 14th edition. J G Collee, T J Mackie, J E McCartney, pg.151 - 154.

11. Harish Naik, Anupama Devi, Sudha Mj. Pattern of Antibiotic prescription in Urinary Tract Infection. Indian J Pharm Pharmacol 2016;3(2);59-62.

12. N Subedi, S Pudasaini. Bacteriological profile and antibiotic sensitivity pattern in patients with Urinary tract infection.Home. J Pathol Nepal 2017;7(1). DOI: http://dx.doi.org/10.3126/jpn.v7i1.16910

13. Beyene G, Tsegaye W. Bacterial Uropathogens in Urinary Tract Infection and Antibiotic Susceptibility Pattern in Jimma University Specialized Hospital, Southwest Ethiopia. Ethiopian J Health Sci 2011;21(2):141-46.

14. R Nalini, J Ezhil Ramya, B Meenakshi, N Palniappan, and S Poongodi. Recent Sensitivity Pattern of Escherichia Coli in Urinary Tract Infection. Journal of Microbiology and Biotechnology.

15. Shalini, Joshi MC, Rashid MK, Joshi HS. Study of Antibiotic Sensitivity Pattern in Urinary Tract Infection At a Tertiary Hospital. Abstracts NJIRM 2011;2(3):43-6. 2. July- September eISSN: 0975-9840 pISSN: 2230 9969.

16. Singhal A, Sharma R, Jain M, Vyas L. Hospital and Community Isolates of Uropathogens and their Antibiotic Sensitivity Pattern from a Tertiary Care Hospital in North West India. Ann Med Health Sci Res 2014;4(1):51-6. doi:10.4103/2141-9248.126611.

How to cite this article: Agarwal R., Pandey G. Bacteriological profile in UTI Cases with special reference to Amikacin sensitivity in a tertiary Care centre in Western UP. Int $J$ Med Microbiol Trop Dis 2018;4(4):218-21. 